\title{
Editorial
}

\section{Dos años de ajuste estructural: ¿hacia dónde vamos?}

Con la llegada del gobierno de ARENA en junio de 1989, en El Salvador se han producido rápidos cambios en la gestión económica y en la orientación global de la economia. Estos cambios han afectado tanto el manejo de la políticas macro y microeconómicas como el marco institucional dentro del que se desenevuelve la actividad económica. Los cambios han estado enmarcados dentro del programa de ajuste estructural y de la nueva estrategia de crecimiento hacia afuera, impulsada con mayor impetu por el actual gobierno. La filosofia que subyacería a ellos es que el requisito de una asignación "óptima" de los recursos que exigiría un crecimiento económico acelerado, sólo puede ser logrado mediante la libre interacción de las fuerzas del mercado. De ahi que muchos de los cambios que han tenido lugar suelan resumirse en una sola frase: liberalización económica.

A dos años de haber entrado al ajuste estructural es pertinente tratar de hacer un breve balance sobre lo que hasta ahora se habria logrado. Aunque, dada la naturaleza del programa, dos anos no es mucho tiempo para juzgar con contundencia sus resultados, si es un buen tiempo para valorar, a partir de las principales tendencias observadas y a partir del contenido del programa, hacia dónde nos conducimos. De no encontramos en la dirección correcta, también es importante sefialar al menos algunos elementos generales que nos ayuden a identificar una senda que sea al menos más adecuada. En nuestro balance evaluativo trataremos tres puntos globales: la estabilización económica, el crecimiento y la problemática económico-social. 


\section{La establlización económica}

Con cierta impresición, puede decirse que uno de los componentes del programa de ajuste esiructural impulsado desde 1989 era la estabilización económica. Por ello habría de entenderse, fundamentalmente, la reducción de la inflación al menos al nivel de la inflación mundial, y la disminución de la brecha extema a niveles compatibles con las entradas netas de capital en el largo plazo. En la visión de las actuales autoridades económicas, para ello sería crucial la elimación, o drástica disminución, del déficit fiscal, asi como el ejercer un control mucho más estricto sobre los agregados monetarios (crédito interno y oferta monetaria). De cara a la brecha externa, también se requerirla de una devaluación de la moneda.

Aunque, en realidad, en los planes origninales del gobierno la estabilización económica debería de ser una etapa previa a la implementación del ajuste estructural propiamente, en la práctica ambos procesos se han impulsado más o menos de manera simultánea. Esto no ha sido del todo sorprendente dado que los desequilibrios macroeconómicos en El Salvador, aunque importantes, no eran alarmantes, sobre todo si se les comparara con los que han registrado otros paises latinoamericanos.

En la agenda de las autoridades económicas salvadorenas, se esperaba que la tarea de estabilización estuviera completada para diciembre de 1990. En general, puede afirmarse que este objetivo no ha sido cumplido, no sólo porque llegó diciembre y la economía no se estabilizó, sino también porque ha transcurrido medio ano más y los desequilibrios macroeconómicos, en términos generales, han tendido a cierta profundización con relación a su estado antes de junio de 1989. En el caso de la inflación, aunque tendencialmente pudiera estar apuntando hacia la baja, en estos dos anos no se habría sino evitado su agravamiento. En efecto, al igual que en los últimos dos anos de la gestión económica demócrata cristiana, en los dos primeros años de la actual gestión, la inflación se ha mantenido en alrededor del $20 \%$ anual*. Distinto es el caso del desequilibrio externo: el saldo de la balanza de bienes y servicios como proporción del producto interno bruto (PIB) se habría incrementado en más o menos 4 puntos porcentuales (de 8 a $12 \%$ aproximadamente), y la situación de balanza de pagos sigue siendo viable gracias a los cuantiosos volumenes de remesas familiares $y$, en menor medida, a la ayuda oficial. Todo esto a pesar de que el

(") A menos que se indique de otro modo, todos los datos aquí citados tienen como fuente el Banco Central de Reserva. 
déficit fiscal estarla tendiendo a una disminución bastante significativa (como proporción del PIB y en términos absolutos); a pesar de que se ha ejercido un control más estricto sobre los agregados monetarios, $y$, en el caso del sector externo, a pesar de que la moneda se ha devaluado en aproximadamente un $60 \%$.

La cuestion fiscal merce una mención especial. El problema va, en general, más allá de la mera estabilización macroeconómica. En el marco de una estructura de gastos bastante rigida y orientada en buena medida hacia la guerra, y de una sistema tributario también bastante rigido y poco eficaz, la disminución del déficit fiscal no ha estado en modo alguno exenta de costos, de costos que no sólo vuelven más deplorable la situación social existente, sino que también van en contra del crecimiento en el mediano y largo plazo. En efecto, el déficit se ha disminuido recortando drásticamente los gastos en capital flsico, y también en capital humano (salud, educación, asistencias social, etc.). Las consecuencias de esto de cara al crecimiento no requiren de mayor explicación.

Vinculado estrechamente a ello estaría el problema de las recaudaciones tributarias. Parece haber un consenso bastante amplio, del que también participan inclusive los organismos financieros intemacionales (especialmente el Banco Mundial), de que los ingresos tributarios en EI Salvador deben incrementarse sustancialmente. La política tributaria del gobierno, sin embargo, estarla moviéndose en otra dirección, particularmente en su componente de reformas a los distintos impuestos directos. Si bien en 1990 las recaudaciones crecieron notablemente, ello estuvo más vinculado al crecimiento de la economia y a una aparente mayor disposición de los sectores económicamente privilegiados a prestar una mayor colaboración al actual gobierno, asi como al incremento de los ingresos por el danino impuesto de timbres, que a cambios saludables operados en el sistema tributario.

Inspirada en la economía del lado de la oferta, la parte de la reforma tributaria en marcha que tiene que ver con los impuestos directos, buscaría incrementar la inversión, mediante la reducción de impuestos a los sectores de más altos ingresos (próximamente habrá refomas nuevas e importantes, especialmente a los impuestos sobre la renta - personal y de sociedades). Sin embargo, existen serias dudas, fundadas tanto en argumentos teóricos como empíricos, de que tal mecanismo lafferiano pueda ser eficaz de cara al fomento de la acumulación de capital. Las dudas se vuelven aún mayores en virtud del sesgo pro-consumista de la reforma comercial en marcha (ver infra). Por otra parte, parecen haber pocas dudas en cuanto a la capacidad 
de la reforma para menoscabar las recaudaciones y sobre todo para acabar con la escasa equidad que el actual sistema tributario conserva. Esto no signifca que el sistema tributario vigente antes de 1989 era perfecto y no requerla de ninguna readecuación. El sistema tenla ciertamente defectos que afectaban su eficiencia, asl como su eficacia de cara a su capacidad de recaudación. El punto es que en un pals como El Salvador no debe intentarse resolver esos defectos a costas de las recaudaciones mismas y de la equidad tributaria.

Volviendo al problema de la estabilización, dadas las medidas de liberalización impulsadas y el marco socio-político en que la economía se ha desenvuelto, el haber puesto un freno a ciertos desequilibrios macroeconómicos no carece totalmente de méritos, aunque algunos de los instrumentos usados no son los más adecuados. Hay que considerar que algunas de esas medidas de liberalización han entrado en claro conflicto con los objetivos de estabilización: en el caso de la inflación, la liberalización masiva de precios en 1989, los frecuentes ajustes a las tarifas de algunos servicios públicos (a la electricidad especialmente), la devaluación, etc. no han vuelto sino más diffcil la tarea de reducirla. En este contexto, la tarea de disminuir la inflación podla muy bien pasar por crear una mayor inflación en el corto plazo, como ha sido el caso hasta ahora. En el caso del sector externo, la agresiva, acelerada e indiscriminada liberalización de las importaciones no ha hecho sino contrarrestar los efectos de la devaluación. Del mismo modo, la presencia del conflicto armado ha contribuido a que, en algunas coyunturas (finales de 1989, por ejemplo), las riendas sobre la situación fiscal y sobre los agregados monetarios se aflojen considerablemente.

Ahora bien, pareciera que en el caso de la inflación, hay cierta tendencia -aunque no del todo clara o sistemática- a su reducción. Puesto que no estamos ante un gobierno interesado en redistribuir el ingreso mediante mecanismos salariales en realidad no to está prácticamente mediante ningún mecanismo- el que esta tendencia se afiance dependerá en buena medida tanto del manejo fiscal y monetario-crediticio que se haga, como de la evolución que sigan el tipo de cambio y la inflación mundial. La variable más frágil entre estas quizá sea la fiscal, aunque en la medida en que el gobierno siga teniendo el poder frente a las instancias de la sociedad civil más afectadas- para recortar el déficit mediante la reducción del gasto, la situación pudiera ser manejable. En cambio, si la reducción del gasto no puede continuar y no se realiza una seria reforma tributaria (incluyendo la mejora de los mecanismos institucionales de la administración tributaria, a fin de reducir la evasión), tendiente a incrementar 
los ingresos tributarios, el problema fiscal puede echar por tierra los esfuerzos de estabilización. Como hemos sugerido arriba, en nuestra opinión, la clave no está en una mayor contracción del gasto, sino fundamentalmente en la elevación de las recaudaciones.

En términos macroeconómicos, el panorama en el sector externo no es menos complicado. Si bien por hoy el déficit comercial no se ha traducido en un drenaje acelerado de reservas internacionales (éstas más bien han aumentado), gracias fundamentalmente a las transferencias $y$, en menor medida, al incremento del endeudamiento externo, asl como a 10 que parecieran ser intentos de repatriación de capitales, la situación es bastante frágil, y pudiera cambiar a no muy largo plazo. En efecto, de continuarse con la actual política de sector externo y de no mejorar sustancialmente el desempeno exportador de la economla, la situación pudiera tornarse inviable desde una perpsectiva macroeconómica, pues no puede esperarse que los flujos de ayuda oficial continúen por mucho tiempo, ni que las remesas familiarse sigan creciendo o se mantengan a los actuales niveles de manera indefinida.

Ahora bien, las implicaciones de la situación del sector externo van más allá de su incidencia en términos de estabilidad macroeconómica. Una dimensión adicional importante que tiene que ver con tal situación lo constituye las caracteristicas que pueda ir asumiento la estructura productiva salvadorena. Este sería tanto más el caso en cuanto que en la actual estrategia económica la política sectorial tiene un rol prácticamente insignificante. En la última década se habria observado una tendencia a la desindustrialización (el mal holandés) con relación a la década de los setenta, sin que ésta sea compensada por un aumento de la importancia relativa del sector agropecuario en el producto total. La contrapartida de este fenómeno es un crecimiento de los servicios y en general de los sectores que producen bienes no comerciados internacionalmente, en los cuales el crecimiento de la productividad suele ser menos rápido y el progreso técnico más diffcil de incorporar. De esta manera, de continuarse con la actual política de sector externo, sería el crecimiento económico el que se llegaria a sacrificar, al menos parcialmente. Esto es ya de suyo un problema grave. Pero, además, las implicaciones volverian, mediante un proceso de retroalimentación, sobre la viabilidad en el largo plazo del mismo sector externo.

\section{El crecimiento económico}

En to que se refiere al ajuste estructural propiamente, como se sabe, esta es una estrategia de crecimiento: su objetivo fundamental es 
acelerar el crecimiento económico sostenible. Dados los esfuerzos de estabilización de corto plazo y la naturaleza de las medidas propiamente de ajuste estructural, este es un programa al menos de mediano plazo. En este sentido, los resultados de crecimiento en los últimos dos anos (conto plazo) deben ser juzgados teniendo en cuenta esos elementos. Por razones diferentes, también debe considerarse el entorno político en que el programa se desarrolla.

Existen algunas dificultades para evaluar el desempeño de la economía en términos de crecimiento en estos dos primeros años de gestión de ARENA. Dado que lamentablemente en El Salvador sólo existen datos anuales de producción, no podemos juzgar exactamente esos dos anos en términos de crecimiento. Para ello se necesitarla datos semestrales, que no existen (el IVAE resulta menos confliables que las cuentas de producción). Así, no se cuenta con indicadores adecuados para evaluar debidamente el primero y el último semestre de ARENA, y estamos obligados a centrarnos en lo que sucedió en 1990.

A juzgar por la estadisticas oficiales, en 1990 la economía salvadorefia habría experimentado el mayor ritmo de crecimiento desde $1978(3.4 \%)$. Sin embargo, más que a una excelente gestión económica, este crecimiento se debió en buena medida a las bondades de la naturaleza, es decir, a que se tuvo un buen clima, que favorecib a la actividad agrícola. Este sería el principal factor en la explicación del crecimiento de $7.4 \%$ del sector agropecuario. La industria manufacturera habria crecido por debajo de la tasa expansión global de la economia, de manera que el lento proceso de desindustrialización aparecido en el decenio pasado habria continuado; mientras que el importante sector construcción, por su parte, experimentó su mayor contracción (12.8\%) en los últimos 10 anos. Por el lado de la "demanda", particularmente preocupante sería la drástica contracción (más de 13\%) de la formación de capital fijo, en un pais que tras una década de guerra ha sufrido un agudo deterioro de su base de capital. Las implicaciones de esto para el crecimiento futuro son obvias.

En suma, el crecimiento se habria distribuido de manera muy desigual entre los distintos sectores productivos y componentes de la demanda. Algo similar estaría sucediendo con su distribución entre los distintos sectores sociales, como veremos más adelante. Por otra parte, teniendo en cuenta el camino que debemos recorrer simplemente para volver a los niveles de ingreso por habitante de 1978, la tasa de crecimiento de $3.4 \%$ resulta sumamente modesta e insuficiente. Sin embargo, tomando en cuenta las limitaciones que impone el contexto político-militar existente y los trastornos que no deja de ocasionar el 
acelerado proceso de liberalización, quizá no se pueda pedir mucho más, y tal crecimiento puede resultar incluso increible para muchos.

Al margen de las desigualdades sectoriales e insuficiencias del crecimiento logrado, es también de suma importancia preguntarse sobre las bases que lo sustentan. En general, desde una perspectiva de mediano y de largo plazo, puede afirmarse que tales base son bastante débiles. Como se senaló arriba, la estretegia económica en marcha descansa en gran medida en un crecimiento liderado por las exportaciones, y muy especialmente por las exportaciones de origen agrlcola. Con tal estrategia en mente, en los últimos dos anos han tenido lugar una serie de cambios en el régimen de política económica. Dentro de ellos, tendría especial relevancia la reforma comercial (liberalización del comercio exterior) que se viene impulsando desde 1989. El objetivo de esta reforma seria avanzar hacia la configuración de un régimen comercial neutral, es decir, que elimine el sesgo en contra de las exportaciones.

Independientemente de si es o no el modelo extrovertido el más viable para El Salvador, hay serias dudas de que un crecimiento que descanse fundamentalmente en las exportaciones agricolas tradicionales o no tradicionales pueda ser lo más adecuado en este país. Las dudas se fundan, por una parte, en lo que esta opción significa en términos de un crecimiento alternativo fundado en un desarrollo industrial, teniendo en cuenta las mayores posibilidades de incorporación de progreso técnico en las actividades manufactureras, la tendencia de esta a la generación de mayores externalidades, la mayor elasticidad ingreso de las manufacturas, etc.; es decir, teniendo en cuenta las mayores ventajas comparativas dinámicas asociadas con la industria manufacturera, en especial con ciertas actividades dentro del sector.

Por otra parte, está también la restricción que la pobre dotación de recursos naturales que El Salvador posee, y su estrechez territorial, imponen a la sutentabilidad del crecimiento de las exportaciones agrlcolas. Esto es tanto más importante si se tiene en cuenta las diferentes racionalidades económicas imperantes en el agro y la competencia por el uso del suelo derivada de ellas, y la posible ventaja comparativa de que pueden gozar ciertos productos que prioritariamente se consumen internamente. Todo esto no significa que las exportaciones agricolas no tradicionales no puedan expandirse con cierto dinamismo y llegar a representar un parte importante del total de exportaciones, sobre todo si el progreso técnico llega a tales actividades. El problema radica en la sustentabilidad de su crecimiento en el tiempo. 
Ahora bien, respecto a las posibilidades de un desarrollo industrial en este marco, no se trata de que no se puedan impulsar simultáneamente los desarrollos agricola e industrial, es decir, de que su fomento simultáneo sea inevitablemente incompatible. El verdadero problema radica en la escasa importancia que, al parecer deliberadamente, se le otorga al desarrollo industrial dentro de la actual estrategia. Esto es particularmente evidente si se tiene en cuenta que, a dos anos de iniciada la estrategia, no se ha definido ningún tipo de política industrial. El problema se vueleve más grave si se considera la amenaza que el proceso de apertura externa significa para el sector industrial, de no impulsarse una reconversión industrial deliberada y sistemática. En efecto, dada la naturaleza de la reforma comercial en marcha, la ausencia de una reconversión industrial y más en general de una política industrial, no puede conducirnos sino a la destrucción de una buena parte de nuestra actual base industrial; es decir, a una mayor desindustrialización.

Esta situación contrasta con el mayor interés que se muestra hacia el sector agropecuario, en particular hacia las actividades no tradicionales de exportación. Aqui si ha habido una polftica sectorial mucho más clara (ejemplo de ello serían los esfuerzos que viene realizando DIVAGRO, de FUSADES). Naturalmente, no es que no deba existir una política agricola y si una política industrial. Teniendo en cuenta nuestro pobre desarrollo agricola, el contar con una polltica de esa naturaleza es imperioso, aunque no debería reducirse únicamente a las actividades de exportación. Pero, igualmente importante, es una política industrial.

Es conveniente recordar que el modelo de crecimiento liderado por las exportaciones impulsado por los famosos palses del sudeste asiático - lodos ellos pobres también en recursos naturales- que acá se quiere imitar, no se ha fundado en cualquier tipo de exportaciones o en cualquier estructura productiva, sino en la exportación de manufacturas y un desarrollo industrial acelerado. (Por cierto, lejos de ser resultado del laissez faire, tal desarrollo ha sido impulsado por una polftica industrial deliberada y sistemática, cuya formulación, orientación e implementación ha sido obra de la intervención estatal). Por otra parte, las exportaciones agricolas de esos palses, como la importancia de su sector agropecuario, son realmente negligibles, sin que por ello el desarrollo agricola haya sido en general sacrificado (Singapur, que prácticamente carece de agricultura, serla una excepción).

Por otra parte, tampoco cabe esperar mucho en términos de acumulación de capital del paquete de política económica que viene siendo 
implementado. Arriba hemos senalado las dudas sobre la eficacia a este respecto de medidas fundadas en la economla del lado de la oferta. Estas dudas son reforzadas por el hecho de que la reforma comercial en marcha conlleva un sesgo en contra de la acumulación. En efecto, al disminuir significativamente los aranceles a todo tipo de bienes de consumo -en el contexto de represión al consumo de bienes importados que estuvo vigente por largo tiempo- $e$ incrementar también significativamente los aranceles a los bienes de capital, la reforma comercial no hace sino estimular el consumo. (Esto al margen de todo lo que el ahorro financiero pueda responder positivamente ante el incremento de las tasas reales de interés). En este contexto, resulta aún más ilusorio esperar que el mayor ingreso disponible con que la reforma tributaria deja a los grupos de más altos ingresos pueda ser destinado a fines de acumulación, y que con ello la economla pueda crecer a un ritmo más acelerado. Es probable que, una vez finalizado el conflicto armado, efectivamente la economia crezca más, pero es muy poco probable que esto se deba a la implementación de tales reformas de política económica. Esto no significa que tales reformas no tendrian de ese modo importancia alguna, pues está su impacto redistributivo adverso, que no carece de trascendencia.

\section{El problema económlco-soclal}

Por último, pero no por ello menos importante, está el problema social. Como se deduce de lo hasta ahora expuesto, tanto la polliticas de estabilización como las de crecimiento económico que vienen siendo impulsadas conllevan grandes costos sociales. En efecto, medidas de estabilización como la devaluación y la reducción del déficht fiscal mediante el recorte del gasto - para citar las que tienen un impacto más fácilmente visible - tienen costos en términos de redistribución del ingreso, desempleo, disminución del gasto social y por tanto del bienestar de algunos grupos, etc. Asimismo, medidas vinculadas más directamente a la problemática del crecimiento, como la elevación de las tasas de interés, la apertura externa, la reforma tributarla, etc. también acarrean costos sociales en términos capacidad productiva instalada, desempleo, equidad (o reducción del gasto social, en el caso de lo tributario), etc.

Algunos de tales costos, como hemos sugerido arriba, son realmente innecesarios. Desgraciadamente, sin embargo, sean o no necesarios, están recayendo de manera desproporcionada sobre los sectores más pobres. La evolución de la distribución del ingreso y la pobreza en 1989-90 en el área urbana, que las encuestas de hogares del Ministerio de Planificación (MIPLAN) han detectado, estarla indl- 
cando precisamente éso. Aunque, en principio, los datos para 1989 y 1990 podrian no ser comparables, dado que las encuestas so realizaron en perlodos (meses) distintos, los datos sobre empleo que las mismas ofrecen sugieren que si puede establecerse una comparación - aunque quizá no estricta o exacta- entre los datos de las dos encuestas referidos a distribución del ingreso y pobreza. Los datos oficiales indican que la distribución del ingreso en las áreas urbanas se habria reconcentrando aún más en 1989-90. Los gnupos de más bajos ingresos no sólo habrian visto reducida su participación en el ingreso total , sino también disminuido, incluso en términos nominales, su ingreso absoluto. En efecto, la participación en el ingreso para el primer quintil se habría reducido de 4.59 a 3.94, mientras para el último habría aumentado de 49.22 a 50.29, en poco más de un ano [MIPLAN, Encuesta de hogares de propósitos múltiples Octubre 1988-Febrero 1989, p. 43; GAES-MIPLAN, Informe económico y social (Enero-Septiembre), p. 112]. Asimismo, para el primer quintil, la disminución del ingreso real andaria cerca del $30 \%$, mientras para el último habría aumentado en más del $30 \%$, en el mismo periodo (/bid.). El resultado de ello habria sido un incremento de la pobreza urbana de 55.2\% en 1989 a 63.0\% en 1990 (lbid.).

Aunque, dadas las medidas de ajuste adoptadas, en la ausencia de un programa de compensación social era totalmente previsible este tipo de efectos, no to era necesariamente -al menos no en las dimensiones que los datos anteriores sugieren- bajo la presencia de tal programa, como es el caso de El Salvador. De modo que, o el programa ha sido pobremente disenado e implementado, 0 es insuficiente, 0 ambas cosas. Lo cierto es que hasta ahora ha resultado ineficaz, y su impacto compensatorio no se ha hecho sentir.

En este marco de deterioro social, el crecimiento económico logrado ha servido de muy poco. No ha llegado donde era más urgente que llegara, $y$ ha llegado especialmente donde bien podriamos darnos el lujo de que aún no llegara: a los sectores más pobres y los sectores más privilegiados, respectivamente. Si se acepta que el objetivo de todo proceso de crecimiento deberla ser la elevación del bienestar de la población en general, pero especialmente del bienestar de la población más pobre, el no mal desempeno de la economia en términos de crecimiento ha carecido por hoy de sentido, al igual que los costos en que ha tenido que incurrirse para ello.

\section{¿Hay alguna alternativa?}

El problema que se presenta, en este contexto, es como dar un 
verdadero sentido al crecimiento y a las medidas que han de posibilitarlo. Por definición, una condición imprescindible para ello es que el crecimiento beneficie especialmente a los más pobres. Ahora bien, si se partiera de que para que haya un crecimiento fuerte y sostenido es necesario incurrir en algunos costos, el siguiente problema es cormo distribuir esos costos. Contrario a lo que hasta ahora ha ocurrido, el camino no es obviamente que éstos sean asumidos por quienes tienen menor capacidad de pagarlos. Ahora, ¿debe ser ese necesariamente el punto de partida?, es decir, desde una perspectiva global, y asumiendo la necesidad de un crecimiento fuerte y sostenido, ¿es realmente inevitalbe incurrir en tales costos? De hecho, si la economla se encuentra creciendo no habrla necesariamente por qué hablar de costos: potencialmente, todos podrlamos salir ganando. El ajuste, en ese caso, podrla ser un juego de suma positiva. Si aceptamos la necesidad del ajuste, asl como la percepción de que, dada la dimensión de los desequilibrios macroeconómicos existentes en EI Salvador y la existencia de una abundancia relativa de divisas, no tiene por qué tratarse necesariamente de un ajuste recesivo; y si aceptamos también de que algunas de las medidas hasta ahora adoptadas incrementan innecesariamente los costos del crecimiento, el problema serla hacer del ajuste un juego de suma positiva.

Ahora bien, para que ésto pueda ser factible, la forma en que el actual programa económico se viene implementando debe ser moditicada, asl como partes importantes de su contenido. Al mismo tiempo, nuevas medidas deberlan ser adoptadas. Por diversas razones, el gobierno puede muy bien negarse a esto, como hasta ahora ha hecho. El problema está en que, llegado cierto punto o cierto tiempo, tal negativa no harla sino acarrear mayores costos, costos que amenacen al crecímiento inclusive en el corto plazo, y que de ese modo descarten totalmente la posibilidad de arrivar a un juego de suma positiva.

En efecto, es evidente que cuando una buena parte de estos sectores perdedores se encuentra organizado -como es el caso salvadoreno-, recurriendo a medidas de presión, estos pueden ser capaces de desestabilizar la economla. Asl, la mera forma en que se realizarla la estabilización (macroeconómica) o el ajuste llevaría engendrada una mayor desestabilización económica. Esta situación serla tanto más probable cuanto mayores sean los espacios que se vayan abriendo como resultado de la finalización del conflicto armado (de la firma de un cese de fuego). Ahora bien, parece claro que, al margen de que esto se concretice o no, dada la marcha de la economla de los sectores menos privilegiados, la desestabilización podrla muy bien suceder, aun cuando se logre elevar la tasa de crecimiento de la 
economia (y aún cuando se registren mayores avances en el combate a la inflación). Al igual que lo que hasta ahora ha sucedido, esto llevaria a un juego de suma negativa, sólo que ya no solamente desde una perspectiva social, sino también desde una perspectiva estrictamente económica.

¿Cómo salir de este callejón? Puesto que no este el lugar para tratar con detalle tan complejo problema, sugeriremos solamente algunas condiciones generales que deberlan estar presentes. Una condición indispensable es la concertación social. Esta deberia partir del reconocimiento de la necesidad de un crecimiento fuerte y sostenible en el largo plazo, y de que sus frutos deben beneficiar a todos, aún en el corto plazo. Esto requiere de un cambio de enfoque sobre el manejo del ajuste. No se trata simplemente de postular su necesidad y luego empezar a contraer la demanda o a adoptar drásticas medidas del lado de la oferta, cualquiera que sea el costo de corto plazo, y luego esperar que el mediano o largo plazo compensen esos costos. Debe partirse de que, primero que todo, el proceso en su conjunto debe ser un proceso viable, y de que ello requiere que, si va a haber crecimiento, que no haya perdedores, ni en términos absolutos ni en términos relativos (todo lo contrario) para los menos privilegiados, y al menos no en términos absolutos para los más privilegiados.

Una vez establecido esto, debería pensarse en la adopción concertada del conjunto de medidas que un ajuste de esa naturaleza requiere; es decir, en avanzar concertamente hacia la readecuación o transformación de la actual estretegia económica gubernamental, de manera que los costos que se asocian con el proceso de ajuste sean superados, y al mismo tiempo se sienten bases institucionales firmes a fin de reducir significativamente la pobreza, sin atentar contra la estabilidad y el crecimiento, ni contra el bienestar económico de todos los salvadorenos en el mediano y largo plazo. 